\title{
ON THE HADAMARD TYPE INTEGRAL INEQUALITIES INVOLVING SEVERAL $\varphi-r-$ CONVEX FUNCTIONS
}

\author{
MEHMET ZEKI SARIKAYA, HATICE YALDIZ, AND HAKAN BOZKURT
}

ABSTRACT. In this paper, new integral inequalities of Hadamard type involving several differentiable $\varphi-r$-convex functions are given.

\section{INTRODUCTION}

It is well known that if $f$ is a convex function on the interval $I=[a, b]$ with $a<b$, then

$$
f\left(\frac{a+b}{2}\right) \leq \frac{1}{b-a} \int_{a}^{b} f(x) d x \leq \frac{f(a)+f(b)}{2}
$$

which is known as the Hermite-Hadamard inequality for the convex functions.

In [10] Pearce et. al. generalized this inequality to $r$-convex positive function $f$ which defined on an interval $[a, b]$, for all $x, y \in[a, b]$ and $t \in[0,1]$

$$
f(t x+(1-t) y) \leq \begin{cases}\left(t[f(x)]^{r}+(1-t)[f(y)]^{r}\right)^{\frac{1}{r}}, & \text { if } r \neq 0 \\ {[f(x)]^{t}[f(y)]^{1-t},} & \text { if } r=0 .\end{cases}
$$

We have that 0 -convex functions are simply log-convex functions and 1-convex functions are ordinary convex functions.

Recently, the generalizations of the Hermite-Hadamard's inequality to the integral power mean of a positive convex function on an interval $[a, b]$, and to that of a positive $r$-convex function on an interval $[a, b]$ are obtained by Pearce and Pecaric, and others (see [9]-[13).

For some results related to this classical results, (see[1], [2, [8], [9]) and the references therein. Dragomir and Mond [1] proved the following Hermite-Hadamard type inequalities for the log-convex functions:

2000 Mathematics Subject Classification. 26D07, 26D10, 26D99 .

Key words and phrases. Hermite-Hadamard's inequality, log-convex functions, Logarithmic mean, Cauchy inequality, Minkowski inequality, Young inequality, Hölder's inequality. 


$$
\begin{aligned}
f\left(\frac{a+b}{2}\right) & \leq \exp \left[\frac{1}{b-a} \int_{a}^{b} \ln [f(x)] d x\right] \\
& \leq \frac{1}{b-a} \int_{a}^{b} G(f(x), f(a+b-x)) d x \\
& \leq \frac{1}{b-a} \int_{a}^{b} f(x) d x \\
& \leq L(f(a), f(b)) \\
& \leq \frac{f(a)+f(b)}{2},
\end{aligned}
$$

where $G(p, q)=\sqrt{p q}$ is the geometric mean and $L(p, q)=\frac{p-q}{\ln p-\ln q}(p \neq q)$ is the logarithmic mean of the positive real numbers $p, q$ (for $p=q$, we put $L(p, q)=p$ ).

This paper, except for the introduction, is divided into two sections. In Section 1 , we give the some definitions of $\varphi$-convex functions given by Noor in [3] and [7] and we will give a new definition. By using the new definition is defined in Section 1 , we will give the proof of main theorems in Section 2.

\section{Definitions}

Let $K$ be a nonempty closed set in $\mathbb{R}^{n}$. Let $f, \varphi: K \rightarrow \mathbb{R}$ be continuous functions. First of all, we recall the following well know results and concepts, which are mainly due to Noor and Noor [7] and Noor [3]. In [3] and [7, the following new class of functions are defined by Noor:

Definition 1. Let $u \in K$. Then the set $K$ is said to be $\varphi$-convex at $u$ with respect to $\varphi$, if

$$
u+t e^{i \varphi}(v-u) \in K, \forall u, v \in K, t \in[0,1] .
$$

Remark 1. We would like to mention that the (1) of a $\varphi$-convex set has a clear geometric interpretation. This definition essentially says that there is a path starting from a point $u$ which is contained in $K$. We do not require that the point $v$ should be one of the end points of the path. This observation plays an important role in our analysis. Note that, if we demand that $v$ should be an end point of the path for every pair of points, $u, v \in K$, then $e^{i \varphi}(v-u)=v-u$ if and only if, $\varphi=0$, and consequently $\varphi$ - convexity reduces to convexity. Thus, it is true that every convex set is also an $\varphi$-convex set, but the converse is not necessarily true, see [3, 7] and the references therein.

Definition 2. The function $f$ on the $\varphi-$ convex set $K$ is said to be $\varphi-$ convex with respect to $\varphi$, if

$$
f\left(u+t e^{i \varphi}(v-u)\right) \leq(1-t) f(u)+t f(v), \forall u, v \in K, t \in[0,1] .
$$

The function $f$ is said to be $\varphi-$ concave if and only if $-f$ is $\varphi-$ convex. Note that every convex function is a $\varphi-$ convex function, but the converse is not true. 
Definition 3. The function $f$ on the $\varphi$ - convex set $K$ is said to be logarithmic $\varphi$ - convex with respect to $\varphi$, such that

$$
f\left(u+t e^{i \varphi}(v-u)\right) \leq(f(u))^{1-t}(f(v))^{t}, u, v \in K, t \in[0,1],
$$

where $f()>.0([\underline{3},[5,[7])$.

Now, we will define a new definition for $\varphi-r-$ convex fonctions as follows:

Definition 4. The positive function $f$ on the $\varphi-r$-convex set $K$ is said to be $\varphi-r-$ convex with respect to $\varphi$, if

$$
f\left(u+t e^{i \varphi}(v-u)\right) \leq \begin{cases}\left((1-t)[f(u)]^{r}+t[f(v)]^{r}\right)^{\frac{1}{r}}, & r \neq 0 \\ {[f(u)]^{1-t}[f(v)]^{t},} & r=0\end{cases}
$$

We have that $\varphi-0$-convex functions are simply logarithmic $\varphi$-convex functions and $\varphi-1$-convex functions are $\varphi$-convex functions.

From the above definitions, we have

$$
\begin{aligned}
f\left(u+t e^{i \varphi}(v-u)\right) & \leq(f(u))^{1-t}(f(v))^{t} \\
& \leq(1-t) f(u)+t f(v) \\
& \leq \max \{f(u), f(v)\} .
\end{aligned}
$$

In [5], Noor established following theorem for $\varphi$-convex functions:

Theorem 1. Let $f: K=\left[a, a+e^{i \varphi}(b-a)\right] \rightarrow(0, \infty)$ be $a \varphi-$ convex function on the interval of real numbers $K^{0}$ (the interior of $K$ ) and $a, b \in K^{0}$ with $a<$ $a+e^{i \varphi}(b-a)$ and $0 \leq \varphi \leq \frac{\pi}{2}$. Then

$$
f\left(\frac{2 a+e^{i \varphi}(b-a)}{2}\right) \leq \frac{1}{e^{i \varphi}(b-a)} \int_{a}^{a+e^{i \varphi}(b-a)} f(x) d x \leq \frac{f(a)+f(b)}{2} .
$$

The main purpose of this note is to establish new integral inequalities Hadamard type involving product of two $\varphi-r-$ convex fonctions. Two refinements of Hadamard's integral inequality for $r$-convex functions recently established by Ngoc et. al. are shown to be recaptured as special instances. The method employed in our analysis is based on the basic properties of logarithms and the application of the well known Hölder's integral inequality and Minkowski's integral inequality.

\section{Main Results}

Now, we start with the following our main theorem.

Theorem 2. Let $f: K=\left[a, a+e^{i \varphi}(b-a)\right] \rightarrow(0, \infty)$ be $\varphi-r-$ convex functions on the interval of real numbers $K^{0}$ (the interior of $K$ ) and $a, b \in K^{0}$ with $a<$ $a+e^{i \varphi}(b-a)$ and $0 \leq \varphi \leq \frac{\pi}{2}$. Then

$$
\frac{1}{e^{i \varphi}(b-a)} \int_{a}^{a+e^{i \varphi}(b-a)} f(x) d x \leq\left(\frac{r}{r+1}\right)^{\frac{1}{r}}\left([f(a)]^{r}+[f(b)]^{r}\right)^{\frac{1}{r}} .
$$


Proof. Since $f$ is $\varphi-r$-convex function and $r \neq 0$, we have

$$
f\left(u+t e^{i \varphi}(v-u)\right) \leq\left((1-t)[f(u)]^{r}+t[f(v)]^{r}\right)^{\frac{1}{r}}, r \neq 0
$$

for all $t \in[0,1]$. It is easy to observe that

$$
\begin{aligned}
\frac{1}{e^{i \varphi}(b-a)} \int_{a}^{a+e^{i \varphi}(b-a)} f(x) d x & =\int_{0}^{1} f\left(a+t e^{i \varphi}(b-a)\right) d t \\
& \leq \int_{0}^{1}\left((1-t)[f(a)]^{r}+t[f(b)]^{r}\right)^{\frac{1}{r}} d t .
\end{aligned}
$$

Using Minkowski's inequality (3.2), we have

$$
\begin{aligned}
\int_{0}^{1}\left((1-t)[f(a)]^{r}+t[f(b)]^{r}\right)^{\frac{1}{r}} d t & \leq\left[\left(\int_{0}^{1}(1-t)^{\frac{1}{r}} f(a) d t\right)^{r}+\left(\int_{0}^{1} t^{\frac{1}{r}} f(b) d t\right)^{r}\right]^{\frac{1}{r}} \\
& =\left(\left(\frac{r}{r+1}\right)[f(a)]^{r}+\left(\frac{r}{r+1}\right)[f(b)]^{r}\right)^{\frac{1}{r}} \\
& =\left(\frac{r}{r+1}\right)^{\frac{1}{r}}\left([f(a)]^{r}+[f(b)]^{r}\right)^{\frac{1}{r}} .
\end{aligned}
$$

Thus, it is the required inequality in (3.1). This proof is complete.

Corollary 1. Under the asumptions of Theorem 圆 with $r=1$, the following inequality holds:

$$
\frac{1}{e^{i \varphi}(b-a)} \int_{a}^{a+e^{i \varphi}(b-a)} f(x) d x \leq \frac{f(a)+f(b)}{2} .
$$

Theorem 3. Let $f, g: K=\left[a, a+e^{i \varphi}(b-a)\right] \rightarrow(0, \infty)$ be $\varphi-r-$ convex and $\varphi-s-$ convex functions on the interval of real numbers $K^{0}$ (the interior of $K$ ) and $a, b \in K^{0}$ with $a<a+e^{i \varphi}(b-a)$ and $0 \leq \varphi \leq \frac{\pi}{2}$. Then

$$
\begin{aligned}
& \frac{2}{e^{i \varphi}(b-a)} \int_{a}^{a+e^{i \varphi}(b-a)} f(x) g(x) d x \\
\leq & \left(\frac{r}{r+2}\right)\left([f(a)]^{r}+[f(b)]^{r}\right)^{\frac{2}{r}}+\left(\frac{s}{s+2}\right)\left([g(a)]^{s}+[g(b)]^{s}\right)^{\frac{2}{s}}
\end{aligned}
$$

and

$$
\begin{aligned}
& \frac{1}{e^{i \varphi}(b-a)} \int_{a}^{a+e^{i \varphi}(b-a)} f(x) g(x) d x \\
\leq & \left(\frac{r s}{(r+2)(s+2)}\right)^{\frac{1}{2}}\left([f(a)]^{r}+[f(b)]^{r}\right)^{\frac{2}{r}}\left([g(a)]^{s}+[g(b)]^{s}\right)^{\frac{2}{s}} .
\end{aligned}
$$


THE HERMITE -HADAMARD'S INEQUALITIES FOR $\varphi-r-$ convex FUNCTIONS 5

Proof. Since $f$ is $\varphi-r$ - convex function and $g$ is $\varphi-s-$ convex function $(r>$ $0, s>0)$, then we have

$$
\begin{gathered}
f\left(a+t e^{i \varphi}(b-a)\right) \leq\left((1-t)[f(a)]^{r}+t[f(b)]^{r}\right)^{\frac{1}{r}} \\
g\left(a+t e^{i \varphi}(b-a)\right) \leq\left((1-t)[f(a)]^{r}+t[f(b)]^{r}\right)^{\frac{1}{r}} .
\end{gathered}
$$

Multiplying both sides of (3.5) by (3.6), it follows that

$$
\begin{aligned}
& f\left(a+t e^{i \varphi}(b-a)\right) g\left(a+t e^{i \varphi}(b-a)\right) \\
\leq \quad & \left((1-t)[f(a)]^{r}+t[f(b)]^{r}\right)^{\frac{1}{r}}\left((1-t)[g(a)]^{s}+t[g(b)]^{s}\right)^{\frac{1}{s}} .
\end{aligned}
$$

Integrating the inequality (3.7) with respect to $t$ over $[0,1]$, we obtain

$$
\begin{aligned}
& \frac{1}{e^{i \varphi}(b-a)} \int_{a}^{a+e^{i \varphi}(b-a)} f(x) g(x) d x \\
\leq & \int_{0}^{1}\left((1-t)[f(a)]^{r}+t[f(b)]^{r}\right)^{\frac{1}{r}}\left((1-t)[g(a)]^{s}+t[g(b)]^{s}\right)^{\frac{1}{s}} d t .
\end{aligned}
$$

Using Cauchy Swartz's inequality, we have

$$
\begin{aligned}
& \text { 3.9) } \int_{0}^{1}\left((1-t)[f(a)]^{r}+t[f(b)]^{r}\right)^{\frac{1}{r}}\left((1-t)[g(a)]^{s}+t[g(b)]^{s}\right)^{\frac{1}{s}} d t \\
& \left.\leq \int_{0}^{1}\left((1-t)[f(a)]^{r}+t[f(b)]^{r}\right)^{\frac{2}{r}} d t\right)^{\frac{1}{2}}\left(\int_{0}^{1}\left((1-t)[g(a)]^{s}+t[g(b)]^{s}\right)^{\frac{1}{s}} d t\right)^{\frac{1}{2}}
\end{aligned}
$$

Using Young's inequality $\left(2 a b \leq a^{2}+b^{2}\right)$ for right-hand side of the inequality (3.9), we have

$$
\begin{aligned}
& (3.10)\left(\int_{0}^{1}\left((1-t)[f(a)]^{r}+t[f(b)]^{r}\right)^{\frac{2}{r}} d t\right)^{\frac{1}{2}}\left(\int_{0}^{1}\left((1-t)[g(a)]^{s}+t[g(b)]^{s}\right)^{\frac{1}{s}} d t\right)^{\frac{1}{2}} \\
& \leq \frac{1}{2} \int_{0}^{1}\left((1-t)[f(a)]^{r}+t[f(b)]^{r}\right)^{\frac{2}{r}} d t+\frac{1}{2} \int_{0}^{1}\left((1-t)[g(a)]^{s}+t[g(b)]^{s}\right)^{\frac{2}{s}} d t .
\end{aligned}
$$

Using Minkowski's inequality right-hand side of the inequality (3.10), we have

$$
\begin{aligned}
& \int_{0}^{1}\left((1-t)[f(a)]^{r}+t[f(b)]^{r}\right)^{\frac{2}{r}} d t \\
\leq & {\left[\left(\int_{0}^{1}(1-t)^{\frac{2}{r}}[f(a)]^{2} d t\right)^{\frac{r}{2}}+\left(\int_{0}^{1} t^{\frac{2}{r}}[f(b)]^{2} d t\right)^{\frac{r}{2}}\right]^{\frac{2}{r}} } \\
= & \left(\frac{r}{r+2}\right)\left([f(a)]^{r}+[f(b)]^{r}\right)^{\frac{2}{r}} .
\end{aligned}
$$


Similarly we have:

$$
\int_{0}^{1}\left((1-t)[g(a)]^{s}+t[g(b)]^{s}\right)^{\frac{2}{s}} d t \leq\left(\frac{s}{s+2}\right)\left([g(a)]^{s}+[g(b)]^{s}\right)^{\frac{2}{s}} .
$$

Adding (3.11) and (3.12) and rewriting (3.8), we obtain (3.3).

Now, using Minkowski's inequality for right-hand side of the inequality (3.9), we have

$$
\begin{aligned}
& \left(\int_{0}^{1}\left((1-t)[f(a)]^{r}+t[f(b)]^{r}\right)^{\frac{2}{r}} d t\right)^{\frac{1}{2}} \\
\leq & {\left[\left(\int_{0}^{1}(1-t)^{\frac{2}{r}}[f(a)]^{2} d t\right)^{\frac{r}{2}}+\left(\int_{0}^{1} t^{\frac{2}{r}}[f(b)]^{2} d t\right)^{\frac{r}{2}}\right]^{\frac{1}{r}} } \\
= & \left(\frac{r}{r+2}\right)^{\frac{1}{2}}\left([f(a)]^{r}+[f(b)]^{r}\right)^{\frac{1}{r}}
\end{aligned}
$$

and similarly

$$
\left(\int_{0}^{1}\left((1-t)[g(a)]^{s}+t[g(b)]^{s}\right)^{\frac{1}{s}} d t\right)^{\frac{1}{2}} \leq\left(\frac{s}{s+2}\right)^{\frac{1}{2}}\left([g(a)]^{s}+[g(b)]^{s}\right)^{\frac{1}{s}} .
$$

Writing (3.13) and (3.14) in (3.9), and rewriting (3.8), we get the desired inequality in (3.4). The proof is complete.

Corollary 2. Under the asumptions of Theorem 3 and with $s=r=1$ we have

$$
\frac{1}{e^{i \varphi}(b-a)} \int_{a}^{a+e^{i \varphi}(b-a)} f(x) g(x) d x \leq \frac{([f(a)]+[f(b)])+([g(a)]+[g(b)])}{6}
$$

and

$$
\frac{1}{e^{i \varphi}(b-a)} \int_{a}^{a+e^{i \varphi}(b-a)} f(x) g(x) d x \leq \frac{([f(a)]+[f(b)])^{2}([g(a)]+[g(b)])^{2}}{3} .
$$

Corollary 3. Under the asumptions of Theorem 3 and with $s=r$ and $f(x)=g(x)$, we have

$$
\frac{1}{e^{i \varphi}(b-a)} \int_{a}^{a+e^{i \varphi}(b-a)} f^{2}(x) d x \leq\left(\frac{r}{r+2}\right)\left([f(a)]^{r}+[f(b)]^{r}\right)^{\frac{2}{r}}
$$

and

$$
\frac{1}{e^{i \varphi}(b-a)} \int_{a}^{a+e^{i \varphi}(b-a)} f^{2}(x) d x \leq\left(\frac{r}{r+2}\right)\left([f(a)]^{r}+[f(b)]^{r}\right)^{\frac{4}{r}} .
$$


Remark 2. If we take $g(x)=1$ in Corollary 2 we have

$$
\frac{1}{e^{i \varphi}(b-a)} \int_{a}^{a+e^{i \varphi}(b-a)} f(x) d x \leq \frac{([f(a)]+[f(b)])+2}{6}
$$

and

$$
\frac{1}{e^{i \varphi}(b-a)} \int_{a}^{a+e^{i \varphi}(b-a)} f(x) g(x) d x \leq \frac{4([f(a)]+[f(b)])^{2}}{3} .
$$

\section{REFERENCES}

[1] S. S. Dragomir and B. Mond, Integral inequalities of Hadamard type for Hadamard type for $\log$ - convex functions, Demonstratio 31(1998),354-364.

[2] S. S. Dragomir and C. E. M. Pearce, Selected Topics on Hermite-Hadamard Inequalities and Applications, RGMIA Monographs, Victoria University, 2000.

[3] M. Aslam Noor, Some new classes of nonconvex functions, Nonl.Funct.Anal.Appl.,11(2006),165-171

[4] M. Aslam Noor, On Hadamard integral inequalities involving two log - preinvex functions, J. Inequal. Pure Appl. Math., 8(2007), No. 3, 1-6, Article 75.

[5] M. Aslam Noor, Hermite-Hadamard integral inequalities for $\log -\varphi-$ convex functions, Nonl. Anal. Forum, (2009).

[6] M. Aslam Noor, On a class of general variotional inequalities, J. Adv. Math. Studies, 1(2008), 31-42.

[7] K. Inayat Noor and M. Aslam Noor, Relaxed strongly nonconvex functions, Appl. Math. E-Notes, 6(2006), 259-267.

[8] B.G.Pachpatte, A note on integral inequalities involving two log-convex functions, Math. Inequal. Appl., 7(2004), 511-515

[9] J.E.Pecaric, F. Proschan and Y.L.Tong, Convex Functions, Partial Orderings and Statistical Applications, Academic Press, New York, 1992.

[10] C.E.M. Pearce, J. Pecaric, V. Šimic, Stolarsky means and Hadamard's inequality, J. Math. Anal. Appl. 220 (1998) 99-109.

[11] P.M. Gill, C.E.M. Pearce, J. Pecaric, Hadamard's inequality for $r$-convex functions, J.Math. Anal. Appl. 215 (1997) 461-470.

[12] G.-S. Yang, D.-Y. Hwang, Refinements of Hadamard's inequality for r-convex functions, Indian J. Pure Appl. Math. 32 (2001) 1571-1579.

[13] N.P.G. Ngoc, N.V. Vinh and P.T.T. Hien, Integral inequalities of Hadamard-type for r-convex functions, International Mathematical Forum, 4 (2009), 1723-1728.

Department of Mathematics, Faculty of Science and Arts, Düzce University, DüzceTURKEY

E-mail address: sarikayamz@gmail.com

E-mail address: yaldizhatice@gmail.com

E-mail address: insedi@yahoo.com 